\title{
ANÁLISIS TECNOLÓGICO DE DISTRIBUCIONES ARTEFACTUALES EN LA PERIFERIA SUDESTE DE LA SIERRA BAGUALES (SANTA CRUZ, ARGENTINA)
}

\author{
KAREN B. BORRAZZO* \\ Recibido: 01/01/2008 \\ Aceptado: 05/06/2008
}

\begin{abstract}
RESUMEN
Este trabajo presenta el análisis tecnológico de los conjuntos artefactuales líticos recuperados en superficie en cuatro loci ubicados en las estribaciones orientales de la sierra Baguales (Santa Cruz, Argentina). Las prospecciones realizadas en la localidad permitieron identificar dos fuentes de materia prima lítica, cuyas caracterizaciones se realizan aquí. Asimismo, se informa un nuevo fechado disponible para las ocupaciones humanas en el área.
\end{abstract}

PALABRAS CLAVE: distribuciones artefactuales de superficie, Patagonia, tecnología lítica.

\section{TECHNOLOGICAL ANALISYS OF SURFACE ARTIFACT DISTRIBUTIONS IN THE SOUTHEASTERN END OF BAGUALES RANGE (SANTA CRUZ, ARGENTINA)}

\begin{abstract}
This paper presents the technological analysis of surface lithic assemblages recovered at four loci localized in the southeastern end of Baguales range (Santa Cruz, Argentina). Two lithic raw material sources were identified through the locality survey and they are also described within here. A new radiocarbon date available for human occupations in the study area is informed.
\end{abstract}

KEY WORDS: surface artifact distributions, Patagonia, lithic technology.

El área de estudio corresponde a la periferia sudeste del cordón Baguales, provincia de Santa Cruz (Argentina). La sierra Baguales tiene una longitud de $60 \mathrm{~km}$ y corre en sentido O-E. El sector aquí estudiado se ubica en un ambiente de estepa, entre 300 y 600 m.s.n.m. Este espacio articula dos regiones con abundantes antecedentes arqueológicos: al norte, la cuenca del lago Argentino (Argentina) y al sur, Última Esperanza (Chile). Varios trabajos han comenzado a evaluar el rol del sector sur de Baguales en la conexión de ambas regiones (Borrero y Franco 2000, Borrero 2003, Borrero et al. 2007).

El sector meridional de la periferia de Baguales ha sido trabajado en el sector chileno por Hauthal (1899), Ortiz Troncoso (1972), Prieto (1993-4. Morano et al. 2008) y, más recientemente, por San Román y Morello (1999, 2003). El sector argentino es trabajado desde 1999 por Borrero y colaboradores (Borrazzo 2006a, 2007; Borrero et al. 2006, 2007; Franco y Borrero 2000, Franco et al. 1999).

* CONICET (Departamento de Investigaciones Prehistóricas y Arqueológicas, IMHICIHU). UBA.

Saavedra 15, piso $5^{\circ}$ (1083ACA) Ciudad Autónoma de Buenos Aires, Argentina. Email: kborrazzo@yahoo.com.ar 
La cronología local disponible es de ca. 4500 años A.P. para las evidencias más tempranas de ocupación, de acuerdo a lo fechados obtenidos en los sitios Cerro Castillo 1 (San Román y Morello 2003) y Cerro León 1 (Borrero et al. 2005). En una escala espacial mayor, el sitio Chorrillo Malo 2 (lago Argentino) y Cueva del Medio y Cueva 1 de Lago Sofía en Última Esperanza proveen un marco cronológico más temprano para las primeras ocupaciones en la región, con una antigüedad superior a 9.000 años AP (Franco et al. 2004, Franco y Borrero 2003; Massone y Prieto 2004, Nami 1986a, 1987; Prieto 1991).

Los materiales estudiados aquí fueron recuperados en los campos que pertenecen a la estancia La Verdadera Argentina. Las muestras están compuestas por artefactos líticos provenientes de cuatro loci de superficie. Ellos son: Cerro León 2 (CL2), Cumbre Cerro León 3 (CCL3), Cumbre Alero Este (CAE) y Transectas 6 (T6) (Figura 1 y 2). El conjunto analizado es de 415 artefactos (Tabla 1).

Características generales de la ocupación del Sudeste de Baguales

Diferentes líneas de evidencia han sugerido una utilización logística del sector sudeste de Baguales. La ocupación humana habría sido poco intensa, caracterizándose por la reocupación de espacios puntuales (Borrazzo 2006a, Borrero y Franco 2000, Borrero 2003, Borrero et al. 2006, 2007). Sin embargo, la intensidad de ocupación se habría incrementado con posterioridad a ca. 2000 años A.P. de acuerdo a la información provista por el conjunto lítico del sitio estratificado CL3 (Borrazzo 2006a).

Las materias primas líticas que fueron utilizadas son predominantemente de origen local (sensu Meltzer 1989), más específicamente de disponibilidad inmediata (sensu Civalero y Franco 2003). Otras rocas disponibles regionalmente se encuentran presentes en menores frecuencias, fundamentalmente bajo la forma de instrumentos y núcleos fragmentados y/o agotados, lascas pequeñas (muchas de ellas de reactivación) y/o desechos indiferenciados de reducido tamaño.

Cerro León 1 y 3 (CL1 y 3) son los sitios de la localidad que han provisto material en capa (Borrazzo 2006a, Borrero et al. 2006, 2007). CL1 se localiza al pie de un bloque errático ubicado en la ladera del cerro León, mientras que CL3 es un alero colapsado ubicado en el valle del cerro León, unos $2 \mathrm{~km}$ al SE de CL1 (Figura 1 y 3). Ambos conjuntos comparten sus características tecnológicas generales (Borrazzo 2006a, Borrero et al. 2006). Entre ellas se destacan el predominio de RGFO entre las materias primas utilizadas, la mayor presencia entre los artefactos asociados a momentos tardíos de litologías no identificadas localmente y la mayor explotación, agotamiento y menor tamaño general de estos últimos con respecto a lo observado en artefactos confeccionados en rocas de disponibilidad local. Para el caso de la utilización de las RGFO, Borrero y colaboradores (2006) indican el predominio de una estrategia expeditiva. En CL1 y CL3 se detectó una tendencia a tamaños mayores en los instrumentos depositados en los niveles es-

TABLA 1. Información de los loci muestreados.

\begin{tabular}{|c|c|c|c|c|c|}
\hline Nombre del locus & Tipo de muestreo & \begin{tabular}{|c|} 
Sup. muestreada \\
(m2)
\end{tabular} & $\begin{array}{l}\text { Cantidad de } \\
\text { artefactos }\end{array}$ & $\begin{array}{l}\text { Abundancia } \\
\text { Estimada }\end{array}$ & $\begin{array}{c}\text { Densidad } \\
\text { (artef./m2) }\end{array}$ \\
\hline Cerro León 2 (CL2) & Cuadrícula de recolección (17 x 6 m) & \begin{tabular}{|c|}
$102 \mathrm{~m} 2$ \\
\end{tabular} & 8 & 7 & 0.069 \\
\hline \multirow{2}{*}{$\begin{array}{l}\text { Cumbre de Cerro León } \\
3 \text { (CCL3) }\end{array}$} & $\begin{array}{l}\text { Transecta } 1 \text { Cuadrículas de } \\
\text { recolección }(10 \times 25 \mathrm{~m} 2)\end{array}$ & $250 \mathrm{~m} 2$ & 9 & $37^{*}$ & $0.074^{*}$ \\
\hline & $\begin{array}{l}\text { Transecta } 2 \text { Cuadrículas de } \\
\text { recolección }(10 \times 25 \mathrm{~m} 2)\end{array}$ & $250 \mathrm{~m} 2$ & 46 & & \\
\hline Cumbre de Alero Este (CAE) & Cuadrículas de recolección $(12$ × 25 m2) & $300 \mathrm{~m} 2$ & 35 & 20 & 0.067 \\
\hline \multirow{2}{*}{ Transecta 6} & Transecta 6 superior $(300 \times 10 \mathrm{~m})$ & $3000 \mathrm{~m} 2$ & 260 & $153^{*}$ & $0.024^{*}$ \\
\hline & Transecta 6 inferior $(350 \times 10 \mathrm{~m})$ & $3500 \mathrm{~m} 2$ & 57 & & \\
\hline Total & - & $7817 \mathrm{~m} 2$ & 415 & 217 & 0.028 \\
\hline
\end{tabular}

* Se realizó una única estimación de la abundancia original de la muestra y la densidad de este locus dada la proximidad espacial y la posible proveniencia de los materiales de T2 desde T1 y de T6i desde T6s. 


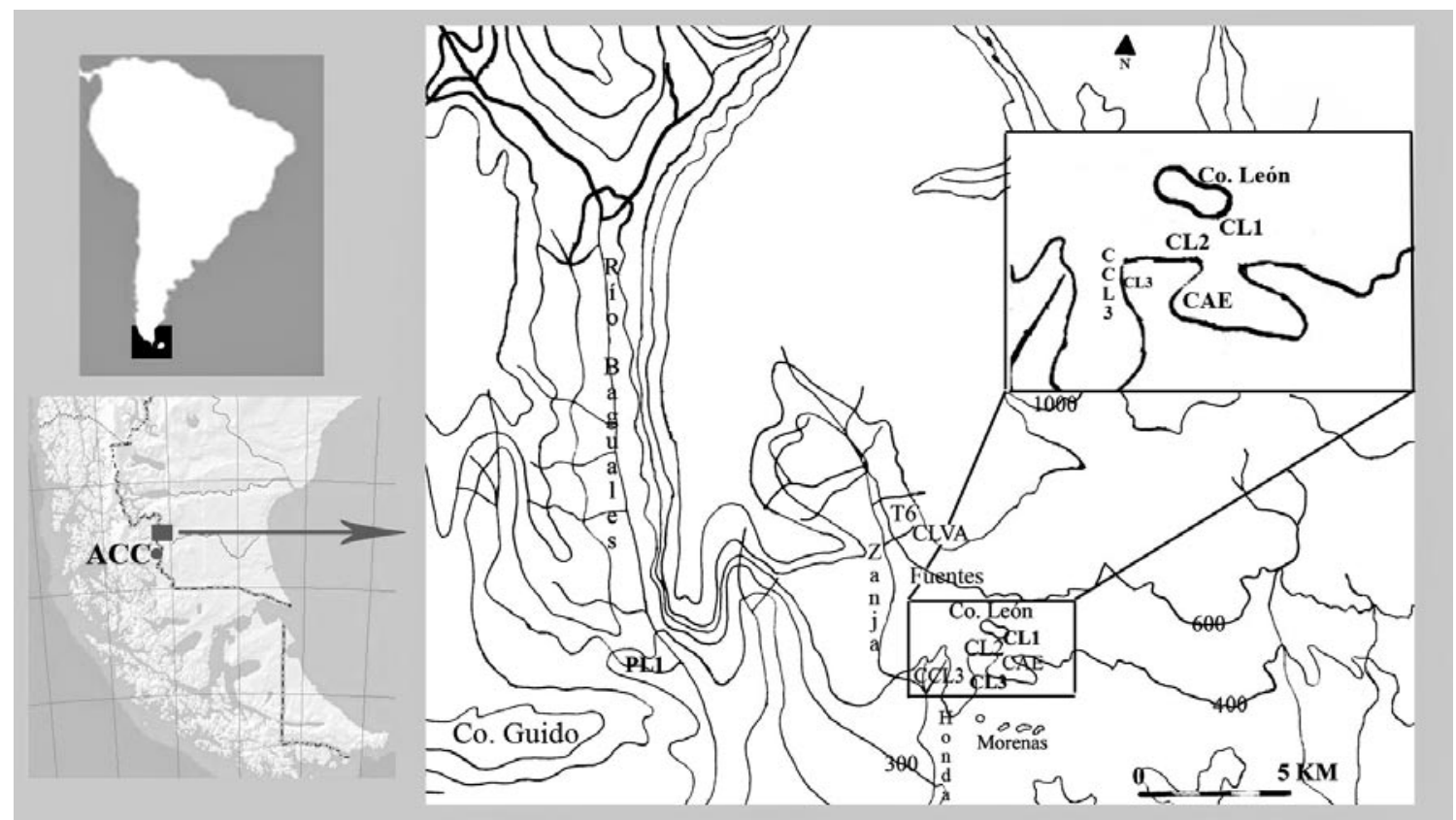

Fig. 1. Área de estudio y sitios mencionados en el texto. CAE: Cumbre Alero Este; CL2: Cerro León 2; CCL3: Cumbre Cerro León 3; CL3: Cerro León 3 (Borrazzo 2006, Borrero et al. 2007; T6: Transecta 6; CLVA: Cueva La Verdadera Argentina; PL1: Puesto Leona 1 (San Román y Morello 1999) y CL1: Cerro León 1 (Borrero et al. 2006 y la localidad Cerro Guido (Prieto 1993-4). En el mapa del sur de la provincia de Santa Cruz se indica la ubicación del sitio Alero Cerro Castillo (San Román y Morello 2003).

tratigráficos más profundos. A diferencia de CL3, el conjunto recuperado en CL1 incluye un núcleo (de dacita) y también evidencias de adelgazamiento bifacial (se recuperó una preforma sobre RGFO). El fechado más antiguo disponible para CL1 es de $4340 \pm 40$ años $C^{14}$ A.P. Por su parte, el estudio de los artefactos recuperados en CL3 permitió delinear diferencias en las estrategias tecnológicas implementadas diacrónicamente en este sector periférico de Baguales (Borrazzo 2006a). Los primeros cincuenta centímetros de la estratigrafía de CL3 - ocupaciones tardías - poseen una mayor densidad artefactual. El conjunto recuperado se caracteriza por la menor frecuencia y tamaño de los instrumentos, así como su mayor fragmentación. En estos niveles se observa un porcentaje mayor de lascas de reactivación y, entre ellas, se destaca la mayor frecuencia de materias primas de calidad muy buena para la talla disponibles regionalmente. Dentro de estas últimas se han identificado calcedonias de variedades macroscópicamente similares a las presentes en conjuntos recuperados al norte de la sierra Baguales (Franco com. pers. 2005). Las características observadas en los artefactos del segmento temporal tardío permitieron sugerir la priorización de una estrategia de equipamiento de los individuos (sensu Kuhn 2004), así como la utilización de toolkits formatizados sobre materias primas de mejores calidad para la talla que las observadas en los niveles inferiores. Se cuenta para estos niveles con una datación radiocarbónica de 1.740 +/- 60 años AP (LP-1669) (Borrazzo 2006a).

Las ocupaciones representadas por el conjunto artefactual recuperado entre 50 y $134 \mathrm{~cm}$ de profundidad en CL3 - ocupaciones tempranas - se habrían caracterizado por la implementación de una estrategia de equipamiento del espacio (sensu Kuhn 2004), dada la presencia de grandes instrumentos enteros con filos activos o reactivables, manufacturados frecuentemente sobre hojas y lascas extraídas de núcleos preparados, todas ellas realizadas sobre materias primas de buena calidad para la talla. La recuperación de ocho percutores de gran tamaño (en todos los casos igual o superior a $385000 \mathrm{~mm}^{3}$ ) es coherente con esta explicación (Borrazzo 2006a). Un nuevo fechado radiocarbónico realizado sobre diáfisis de fémur con marcas de corte recuperado a $84.5 \mathrm{~cm}$ de 

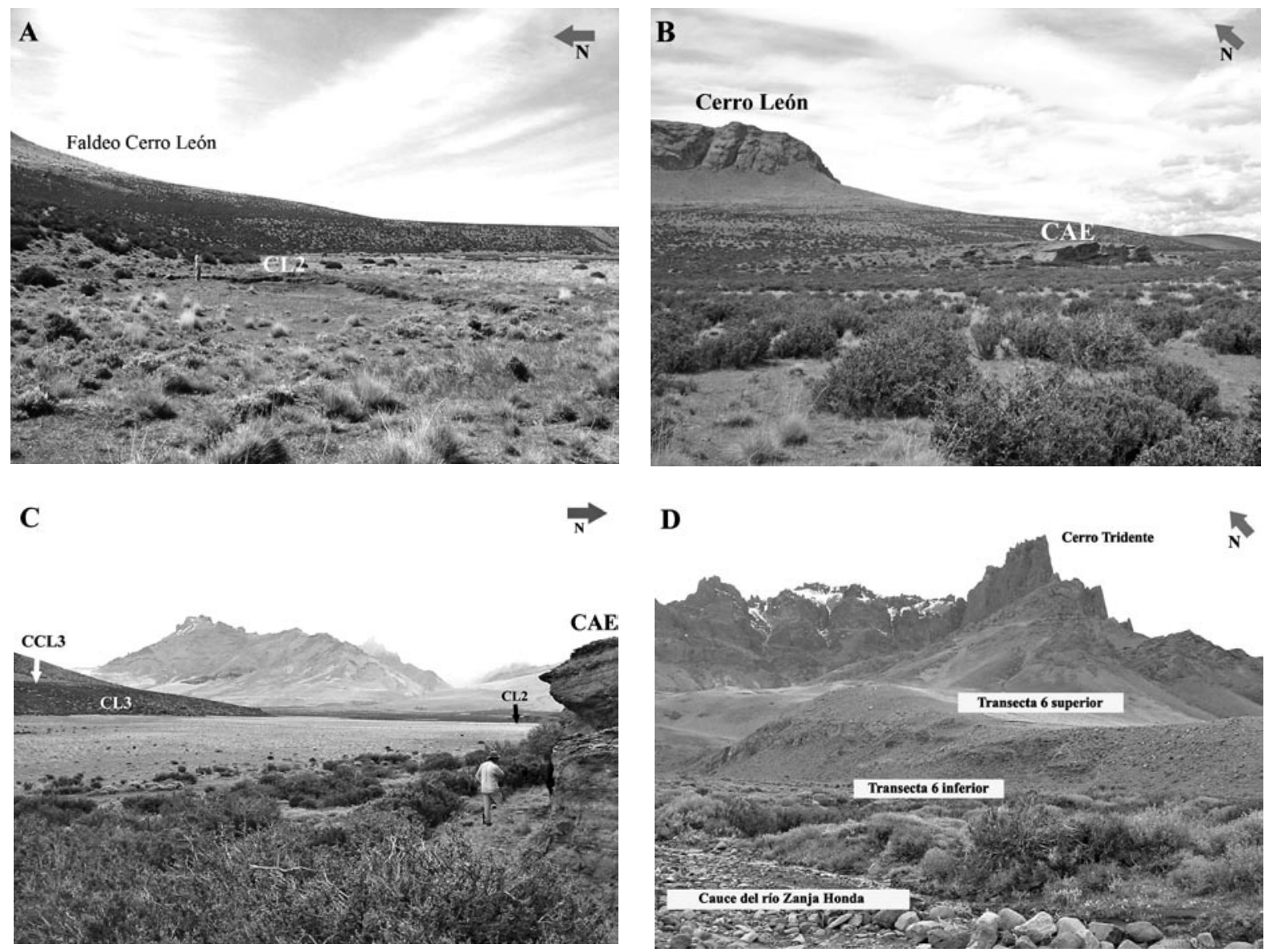

Fig. 2. Fotos de la localidad: A) Cicatriz de erosión donde se localiza CL2; B) CAE; C) Vista panorámica del valle del cerro León y los loci CCL3, CAE y CL2; D) Localización de T6.

profundidad ha provisto una edad de $4370 \pm 50$ años $C^{14}$ A.P. ${ }^{1}$ (GX-32583-AMS). Las evidencias más tempranas para la tecnología de hojas en la localidad provienen de ese nivel.

A continuación se presenta la información obtenida del análisis de las muestras recuperadas en superficie en el área de estudio. Esta caracterización del registro de superficie se suma a la ya disponible (Borrero et al. 2006). Hacia el final de este trabajo, se integran la información arqueológica de superficie y estratigrafía en la discusión sobre el uso de este espacio al Sudeste de la sierra Baguales.

\section{MATERIALES Y MÉTODOS}

Los conjuntos artefactuales estudiados fueron obtenidos a partir de diferentes muestreos de superfi-

1 delta ${ }^{13} \mathrm{C}=-19.7$ per mil. cie practicados dentro de los campos pertenecientes a la estancia La Verdadera Argentina, localizada en la orilla Este del río Zanja Honda, límite internacional con la República de Chile (Figura 1). Los trabajos en el campo que originaron los conjuntos aquí analizados implicaron la recolección total de los artefactos líticos disponibles en la superficie de las unidades muestreadas.

El cálculo empleado para establecer la densidad artefactual de cada conjunto es la suma del MNF (sensu Hiscock $2002^{2}$ ) y los instrumentos, núcleos y desechos indiferenciados enteros. El MNF (o número mínimo de lascas) ha sido calculado por materia

2 MNF (minimum number of flakes) $=$ № lascas enteras + № fragmentos proximales ó distales de lascas (seleccionando de éstos el fragmento más frecuente en la muestra) + № fragmentos longitudinal izquierdos ó derechos (seleccionando de éstos el tipo de fragmento más frecuente en la muestra) (Hiscock 2002). 
prima, para lograr una estimación más ajustada del conjunto original (ver Borrazzo 2004).

La clasificación litológica de las materias primas se realizó comparando macroscópicamente los artefactos con muestras de mano a las que se le han realizado cortes delgados y han sido identificadas microscópicamente por la Dra. María Clara Etchichury (Laboratorio de Petrografía, Museo de Ciencias Naturales "Bernardino Rivadavia"). Para la definición de calidades para la talla, sigo la propuesta de Aragón y Franco (1997). Como ya ha sido observado en otro lugar, entre las materias primas utilizadas existe un conjunto de rocas de origen sedimentario y volcánico cuyas litologías no son identificables macroscópicamente (Borrazzo 2006, 2007; Borrero et al. 2006). Entre ellas se han identificado microscópicamente lutitas, fangolitas y grauvacas; asimismo, ha sido señalada la disponibilidad de basalto en el área (Borrero et al. 2006, Franco y Aragón 2004). Para la clasificación macroscópica de esas materias primas, sigo la propuesta de Charlin (2005), y las incluyo en un grupo denominado "rocas de grano fino oscuras" (RGFO).

El análisis tecno-morfológico ha sido realizado en base a la propuesta de Aschero (1975, 1983), adicionándole las modificaciones propuestas por Franco (2002).

\section{Cerro León 2}

CL2 $\left(069^{\circ} 4.586,43^{\circ} 62.450\right)$ es una cicatriz de erosión localizada en el valle del cerro León, ubicado al NO de CL3 (Figura 1 y 2). En superficie, se observaron pisadas de ovejas. Se recuperaron materiales en superficie y en estratigrafía. En este último caso se realizó un sondeo de $1 \mathrm{~m}$ por $50 \mathrm{~cm}$ de superficie y $50 \mathrm{~cm}$ de profundidad, ubicado en un perfil disponible en un borde de la cicatriz. Se observó abundante materia orgánica a lo largo de toda la estratigrafía (Barberena com. pers. 2004). El material proveniente del sondeo está compuesto por un hueso de oveja y artefactos de RGFO: tres lascas primarias, dos secundarias, tres angulares $y$ un desecho indiferenciado. La muestra de superficie (Tabla 1) está compuesta por ocho artefactos (una lasca primaria, dos lascas angulares, tres núcleos y una lasca de adelgazamiento fracturada en dos partes que pudo ser reparada, sensu Ramos 1993). Las materias primas de la muestra de superficie son en todos los casos RGFO, a excepción de un núcleo de calcedonia color castaño (Munsell 2.5Y $7 / 6$, bright yellowish brown), roca que no ha sido identificada localmente. Una lasca angular remonta (sensu Ramos 1993) sobre uno de los núcleos de RGFO (tamaño del núcleo: 180 por 96 por $59 \mathrm{~mm}$, forma base: nódulo).

La presencia de materiales en estratigrafía y la cercanía de los mismos a la cicatriz de erosión sugieren que la distribución de superficie es resultado del desmoronamiento progresivo del perfil. La presencia de ovejas y el registro del proceso de pisoteo asociado a ellas pueden señalarse como aceleradores del proceso de erosión del depósito sedimentario. La presencia del hueso de oveja en estratigrafía señala el potencial que ofrece este sector para el enterramiento de materiales, situación que ha sido escasamente observada en la localidad (Borrero y Martin, com. pers. 2007).

La similitud macroscópica entre las materias primas de casi la totalidad de la muestra sumada a las relaciones de ensamblaje mencionadas y la alta frecuencia de lascas con corteza, señalarían la realización de actividades de desbaste inicial y extracción de formas base en el lugar. Los núcleos de RGFO son de lascados aislados, y el de calcedonia es discoidal irregular. Este último ha sido retomado (presenta negativos de lascados con diferente intensidad de meteorización). Ningún núcleo está agotado. Se observaron en la muestra talones estallados, naturales, diedros y facetados.

\section{Cumbre de Cerro León 3}

La Cumbre de Cerro León 3 (CCL3, 069을 $4.615,43^{\circ}$ 61.389) es el sector más bajo (ca. 410 m.s.n.m.) del cerro sin nombre al pie de cuya pared se localiza el sitio CL3 (Figura 1, 2 y 3). CCL3 es una franja de $35 \mathrm{~m}$ de ancho que corre paralela a la pared o al talud (dependiendo del sector) del cerro y se encuentra a unos $10 \mathrm{~m}$ sobre el nivel del valle. Su sustrato está formado por un nivel superficial de sedimento arenoso, de espesor variable, constituido en un $90 \%$ por el regolito de la roca de base y el 10\% restante por materiales alóctonos, representados por rodados de rocas volcánicas - preferentemente basalto - y fragmentos de yeso (Etchichury com. pers. 2007). Éste suprayace directamente a la formación de roca sedimentaria del alero CL3 (Borrazzo 2006a). Existen 


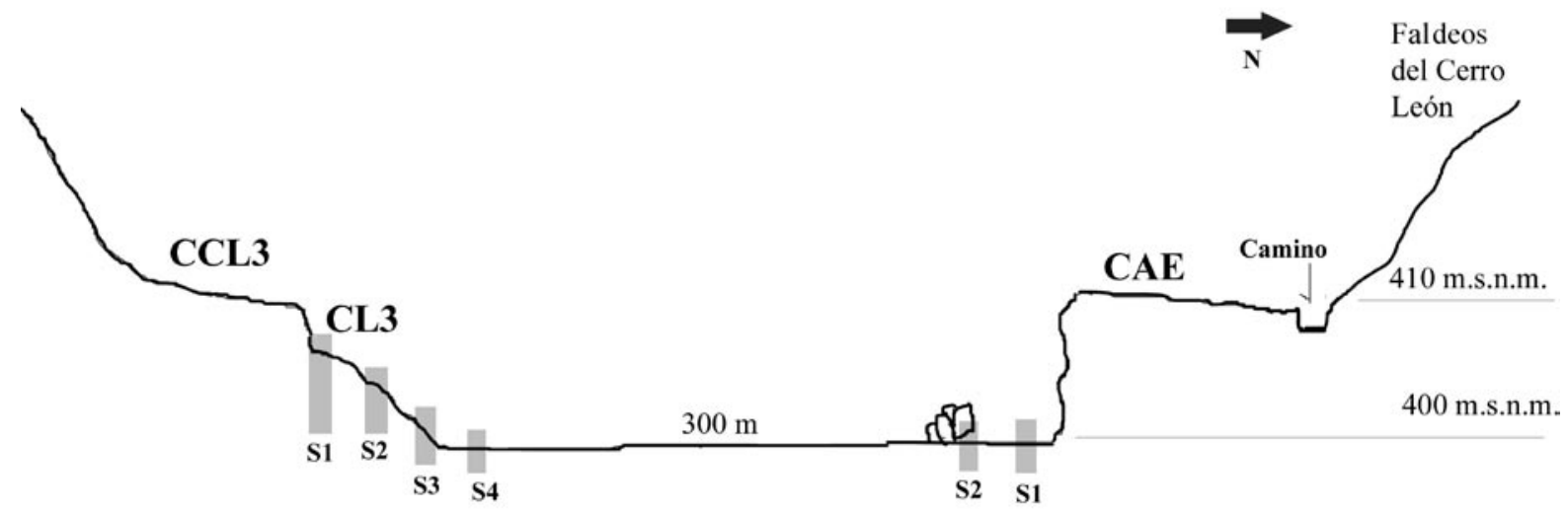

Fig. 3. Corte esquemático del valle de cerro León. Se incluyen la localización del sitio CL3 y los sondeos realizados en el valle.

sectores - cercanos al inicio del talud o al filo del farallón - en que puede observarse la sedimentita expuesta, escasamente cubierta por su regolito. El tamaño de la superficie a muestrear fue 35 por $200 \mathrm{~m}$ (Tabla 1). Para ello se realizaron dos transectas paralelas entre sí, recolectándose en cada una de ellas cuadrículas de $25 \mathrm{~m}^{2}$ con intervalos de $20 \mathrm{~m}$ (10 unidades de recolección por transecta). La primera (T1) se localiza a aproximadamente $15 \mathrm{~m}$ del borde del talud. Seis de las cuadrículas presentaron hallazgos. La segunda transecta (T2) se ubicó paralela a T1, a $10 \mathrm{~m}$ hacia abajo en la pendiente desde T1 y, por lo tanto, muestreó el espacio de CCL3 inmediatamente adyacente al inicio del talud. En T2 siete unidades presentaron hallazgos. La cobertura vegetal de las unidades de recolección en ambas transectas osciló entre un 25 y $75 \%$ de la superficie. En CCL3 no se observó una correlación estricta entre la frecuencia de hallazgos y la cobertura vegetal (hubo casos en que las unidades más vegetadas entregaron abundantes artefactos y viceversa).

La T2 se caracteriza por una mayor frecuencia de unidades de muestreo con pendientes elevadas (40\% posee ángulos de entre 18 y $25^{\circ}$ ) con respecto a lo observado en las cuadrículas de T1 (10\% presenta ángulo de $18^{\circ}$ ). Como se observa en la tabla 1 , la T2 posee una densidad artefactual superior a la de T1. Adicionalmente, en T2 las piezas pequeñas son más frecuentes que en T1, aún cuando su visibilidad es similar. La muestra de T1 no presenta piezas con volúmenes por debajo de los $6500 \mathrm{~mm}^{3}$, mientras que en T2 esos son los más frecuentes (47.2\% de la muestra de T2). Los patrones de distribución espacial de frecuencias y tamaños artefactuales podrían estar señalando la incidencia de la pendiente en la disposición de los artefactos líticos en la superficie de CCL3. Para monitorear este y otros fenómenos, en CCL3 se planteó una cuadrícula experimental en el año 2004. Las primeras observaciones de control realizadas en 2005 registraron deslizamientos horizontales de hasta $49 \mathrm{~cm}$ y se constató que los mayores desplazamientos (pero no los únicos) se realizaron hacia abajo en la pendiente (Borrazzo 2007). Esta información preliminar presenta a CCL3 como un espacio dinámico. Los desplazamientos hacia abajo en la pendiente tendrían el potencial de generar espacios de alta densidad artefactual sobre el talud de CCL3 o en el piso del valle más cercano al farallón. Sin embargo, tres sondeos realizados en el talud de CL3 (Figura 3) mostraron una drástica caída de la frecuencia artefactual desde el espacio en que se planteó la excavación del sitio ( $\mathrm{S} 1$, en Figura 3) (Borrazzo 2006a, Borrero et al. 2007), señalando una baja dispersión de los artefactos. Son necesarios nuevos relevamientos de la pista experimental para calibrar y discutir el rol de los procesos naturales en la conformación del registro arqueológico de superficie y estratigráfico.

Las materias primas más frecuentes en CCL3 son las RGFO (74.6\%). Dacitas, ftanitas y sílices completan la muestra ${ }^{3}$. La composición por tipos artefactuales se informa en la tabla 2.

Entre los desechos sólo unos pocos presentan reserva de corteza (8\%) y predominan las lascas angulares (77.5\%). Se registra la presencia de lascas

3

Se recuperó en una recolección selectiva un fragmento proximal de lascas angular de calcedonia traslúcida con impurezas dispuestas en forma de pintas color moradas. Esta materia prima no ha sido identificada localmente. Aún cuando esta pieza no se incluyó en el presente análisis, se informa su presencia en CCL3. 
TABLA 2. Composición de las muestras artefactuales.

\begin{tabular}{|l|c|c|c|c|c|c|}
\hline & CAE & CL2 & CCL3 & T6 & Total \\
\hline Lascas y desechos indif. & 29 & 14 & 75 & 277 & 395 \\
\hline Núcleos & 1 & 3 & 1 & 3 & 8 \\
\hline Instrumentos* & & & 2 & 7 & 9 \\
\hline Raedera & & & & 8 & 8 \\
\hline Punta burilante & 1 & & 1 & 4 & 6 \\
\hline Lasca c/rastros comp. & 2 & & 1 & 3 & 6 \\
\hline Cuchillo de filo retocado & 2 & & & 1 & 3 \\
\hline Cepillo & $2 *$ & & & 5 & 5 \\
\hline Cortante & & & & 4 & 5 \\
\hline Raspador & & & 1 & 4 & 5 \\
\hline Muesca & & & & 4 & 4 \\
\hline Artef. de format. sum & & & & 2 & 2 \\
\hline Artef. con rastros comp. & & & & & 1 & 1 \\
\hline Filo en bisel abrupto & & & & 1 & & 1 \\
\hline Yunque & & & & 1 & 1 \\
\hline RBO & & & & & 1 & 1 \\
\hline Denticulados & & & & 1 & 1 \\
\hline Cuña & 5 & 0 & 7 & 46 & 58 \\
\hline Total Instrumentos & & & & \\
\hline
\end{tabular}

* Se listan todos los filos identificados en los artefactos. Dado que una misma pieza puede presentar varios filos, la cantidad de instrumentos puede superar el número real de piezas.

** Uno de estos cepillos está compuesto por tres fragmentos remontados (figura 5).

de arista y hojas. Los talones más frecuentes son los lisos, seguidos por los diedros, facetados, naturales y estallados.

Se ha recuperado un núcleo manufacturado sobre un rodado de dacita y una lasca cuya materia prima y corteza son macroscópicamente similares a las del núcleo. Estos dos artefactos son los únicos de CCL3 que tienen evidencias de haber sido reclamados. Aún cuando no se registraron relaciones de remontaje entre estas piezas, las similitudes registradas sumadas a la cercanía espacial de ambos artefactos (4 m) así como la presencia en la muestra de un yunque-percutor sugieren la realización de actividades de extracción de formas base en CCL3.

Cinco de los artefactos recuperados son instrumentos. El grupo tipológico más frecuente es la raedera. En todos los casos han sido confeccionadas en dacita y sílice. Los ángulos medidos en esos filos son $70^{\circ}$ y $80^{\circ}$. Las formas base utilizadas para su manufactura han sido una lasca con dorso natural y una secundaria. Fuera de los muestreos, se identificaron un raspador de sílice y una raedera en dacita gris oscuro, el primero de ellos fragmentado.
Planta de muestreo de CAE

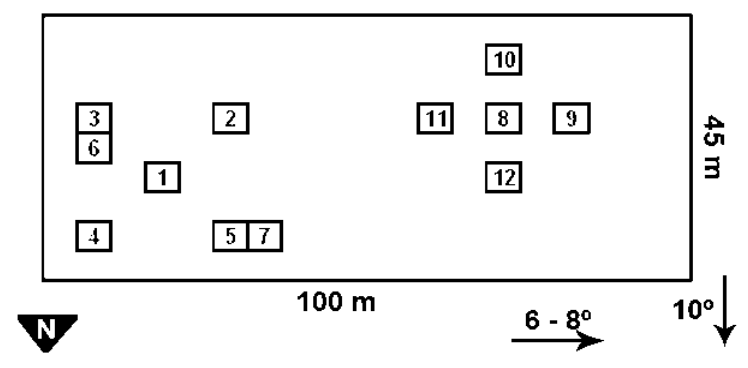

Fig. 4. Planta de muestreos realizados en CAE.

\section{Cumbre de Alero Este}

Se muestreó la cumbre de un alero que mira al SO y se ubica frente al sitio CL3, del que se encuentra separado ca. $300 \mathrm{~m}$ por el valle de Cerro León (Figura 1, 2 y 3). Se trata de CAE (069우 4915, $43^{\circ}$ 61.652), que es la continuación topográfica de CCL3. Al igual que CCL3, está a ca. 410 m.s.n.m. y su superficie está cubierta por el regolito producto de la desintegración de la roca sedimentaria en la que se labra el valle. El sedimento superficial presenta $10 \mathrm{~cm}$ de potencia en el sector más cercano al borde del farallón. Al pie de este último se realizaron dos sondeos de 50 por $50 \mathrm{~cm}$ que alcanzaron los $40 \mathrm{~cm}$ de profundidad y no entregaron material arqueológico en capa (Figura 3).

La superficie sujeta a muestreos ha sido de 45 por $100 \mathrm{~m}$. Se recolectaron dos grupos de cinco cuadrículas (de 5 por $5 \mathrm{~m}$ cada una). El muestreo se iniciaba estableciendo una cuadrícula central, localizándola en el sector donde la densidad de materiales era mayor (Figura 4). Los cuatro muestreos restantes eran distribuidos sistemáticamente a intervalos de $5 \mathrm{~m}$ siguiendo un diseño en cruz. Asimismo, se recolectaron dos cuadrículas $(5$ por 5 $\mathrm{m})$ adicionales dirigidas a recuperar concentraciones de piezas en los espacios inmediatos a dos de las unidades muestreadas sistemáticamente (cuadrículas 6 y 7 en Figura 4).

Las cuadrículas fueron orientadas en sentido N-S. De las 12 cuadrículas planteadas, sólo ocho presentaron hallazgos.

La superficie muestreada presenta una pendiente de $10^{\circ}$ hacia el Norte y entre $6^{\circ}$ y $8^{\circ}$ hacia el Oeste (Figura 4). A diferencia de CCL3, la pendiente baja hacia el camino y no hacia el valle (Figura 3). Se observó que la cubierta sedimentaria era más 


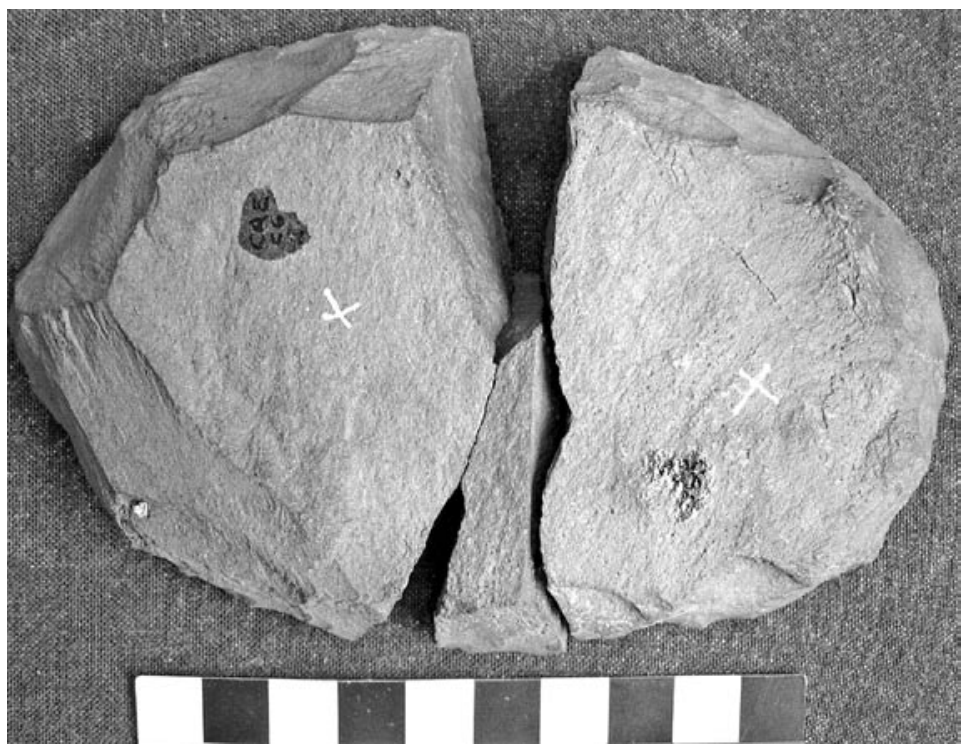

Fig. 5. Cepillo reparado recuperado en los muestreos realizados en CAE.

potente en las cuadrículas ubicadas más abajo en la pendiente N-S de CAE. Adicionalmente, la distribución espacial de tamaños de partículas artefactuales (Borrazzo 2006b) se caracteriza por la presencia de los tamaños inferiores a $40 \mathrm{~mm}$ casi exclusivamente en las cuadrículas 4, 5 y 7, localizadas en el sector más bajo de la pendiente $\mathrm{N}-\mathrm{S}$. Las cuadrículas 4 y 6 se encuentran conectadas por relaciones de ensamblaje (en este caso, reparación sensu Ramos 1993): un fragmento recuperado en la cuadrícula 4 ensambla con dos fragmentos de cepillo de mayor tamaño provenientes de la cuadrícula 6 (Figura 4 y 5). Esto sugiere que las piezas pequeñas habrían reptado con mayor facilidad siguiendo la pendiente N-S en CAE. Por lo tanto, su ausencia en cuadrículas ubicadas en sectores superiores de la pendiente puede responder a factores postdepositacionales. Dadas las características compartidas de CAE con CCL3, la información aportada por las observaciones longitudinales de la pista experimental será relevante para discutir esta hipótesis.

En CAE sólo se recuperaron artefactos en RGFO, a excepción de un cepillo manufacturado en dacita verde, cuyo filo estaba embotado. Entre los desechos predominan las lascas angulares, seguidas por las de arista. Se registra la presencia de lascas de adelgazamiento bifacial y primarias. Los talones más frecuentes son los lisos, seguidos por los diedros, naturales, facetados y estallados.
Se recuperaron siete instrumentos (enteros y fragmentados). Tres de los fragmentos forman parte de un cepillo. Esto totaliza dos cuchillo, dos cepillos y una lasca con rastros complementarios.

Se recuperó un núcleo piramidal irregular de RGFO cuya forma base ha sido un nódulo. Presenta un número mínimo de cinco extracciones, todas ellas con terminación aguda. Su tamaño es $100 \mathrm{~mm}$.

\section{Transecta 6}

Se localizó en un sector cercano al río Zanja Honda, donde hay una pared que presenta aleros de ca. $1.5 \mathrm{~m}$ de altura que miran al O (69우 3077, 43은 63825) (Figura 1 y 2). Allí se plantearon dos transectas de $300 \mathrm{~m}$, paralelas entre sí. La primera de ellas (T6 inferior, T6i), paralela a la base de la pared y la segunda (T6 superior, T6s), sobre la cumbre de los aleros. En ambos casos se realizó un muestreo continuo, utilizando unidades de recolección consecutivas de 10 por $50 \mathrm{~m}$. La homogeneidad ambiental determinó la longitud de las transectas. La dirección de las transectas fue S-N. Se realizaron sondeos en T6i que no aportaron evidencia arqueológica en capa.

La frecuencia de recuperación de artefactos fue mayor en T6s (Tabla 1). Pero ese sector ofrecía mejores condiciones de visibilidad que T6i, donde la vegetación cubría entre un 70 y $90 \%$ de la superficie. 


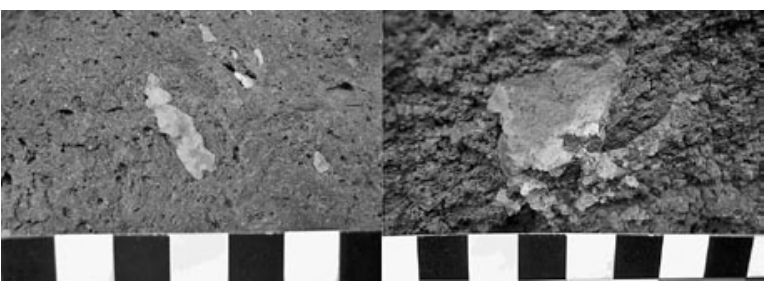

Fig. 6. Calcedonias in situ (pared interna de la cueva La Verdadera Argentina).

Aún reconociendo estas diferencias, es notable la correspondencia entre las unidades con mayores densidades de T6s y T6i (los tres primeros muestreos de cada transecta, es decir, los primeros $150 \mathrm{~m}$ de las mismas). Si se considera la ubicación topográfica (arriba) de T6s cabe plantear como hipótesis que allí se ubicaría el centro de dispersión de los materiales y que, tal vez, los artefactos recuperados en T6i provengan de la cumbre de los aleros (T6s). Esto será evaluado en futuros trabajos de campo en la localidad.

En lo que respecta a la composición artefactual, en la muestra de T6 predominan las lascas angulares, seguidas por las lascas de arista. La mayoría de las lascas (85\%) no presentan reserva de corteza. Los talones lisos son los más frecuentes, seguidos por los diedros, facetados, estallados y naturales. Las RGFO representan el 95\% de la muestra, y el $88 \%$ de los instrumentos, siendo estos valores los más elevados registrados en la localidad. En T6s se recuperaron tres artefactos de calcedonia (dos de ellos macroscópicamente similares a las variedades disponibles en la localidad, ver infra).

\section{Fuentes de materias primas líticas}

Se identificaron dos fuentes de materia prima lítica en los faldeos meridionales del cerro Tridente (Figura 1). La primera de ellas es de calcedonia y se localiza a lo largo del talud de la cueva La Verdadera Argentina (5050237, $72^{\circ}$ 14220). Es una fuente primaria con una distribución dispersa (sensu Nami 1986b, 1991). Se trata de una variedad de calcedonia incolora que se aloja en la matriz volcánica que constituye la roca de caja de la cueva (Figura 6). A medida que la meteorización del cerro avanza, se produce el desprendimiento de fragmentos que incluyen venas o vesículas de calcedonia. Estos fragmentos se encuentran disponibles a lo largo del talud de ca.

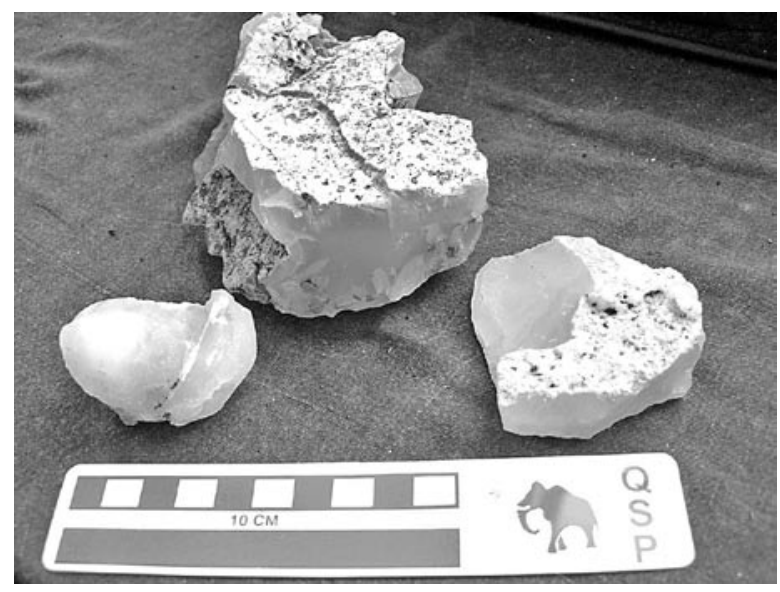

Fig. 7. Nódulos de calcedonia recuperados en el talud de la cueva La Verdadera Argentina.

$1000 \mathrm{~m}$, haciéndose particularmente abundantes en los perfiles que quedan expuestos como resultado de pequeños deslizamientos rotacionales del suelo. La calidad de los nódulos recuperados es variable, de regular a muy buena. Los tamaños y formas de los nódulos de calcedonia de buena calidad para la talla disponibles también son variables y, en muchos casos, requieren de la eliminación de una corteza muy espesa, de calidad regular a mala para la talla (p.e. ver nódulo superior en Figura 7). Se han recuperado ejemplares con cortezas muy delgadas cuyas dimensiones son 57 por 52 por $20 \mathrm{~mm}$ y 50 por 35 por $30 \mathrm{~mm}$ (nódulos inferiores en Figura 7). En esta fuente no se observaron evidencias de trabajo de talla. Como ha sido señalado por varios autores (Borrero et al. 2007, Franco y Aragón 2004) existiría en la localidad un segundo tipo de calcedonia, también incolora y macroscópicamente indiferenciable de la volcánica, pero que es de origen sedimentario. He observado estos nódulos, que se presentan de manera aislada, en las cercanías de los depósitos de drift. De acuerdo a mis observaciones, estos nódulos presentan, en general, mayores tamaños y son más homogéneos en términos de su calidad para la talla (de muy buena a excelente) en comparación con los disponibles en el talud de la cueva La Verdadera Argentina. Se han identificado en superficie núcleos de lascados aislados en la calcedonia de origen sedimentario. El análisis de las muestras artefactuales del área indican un aporte reducido de la calcedonia incolora, macroscópicamente compatible con cualquiera de las variedades 


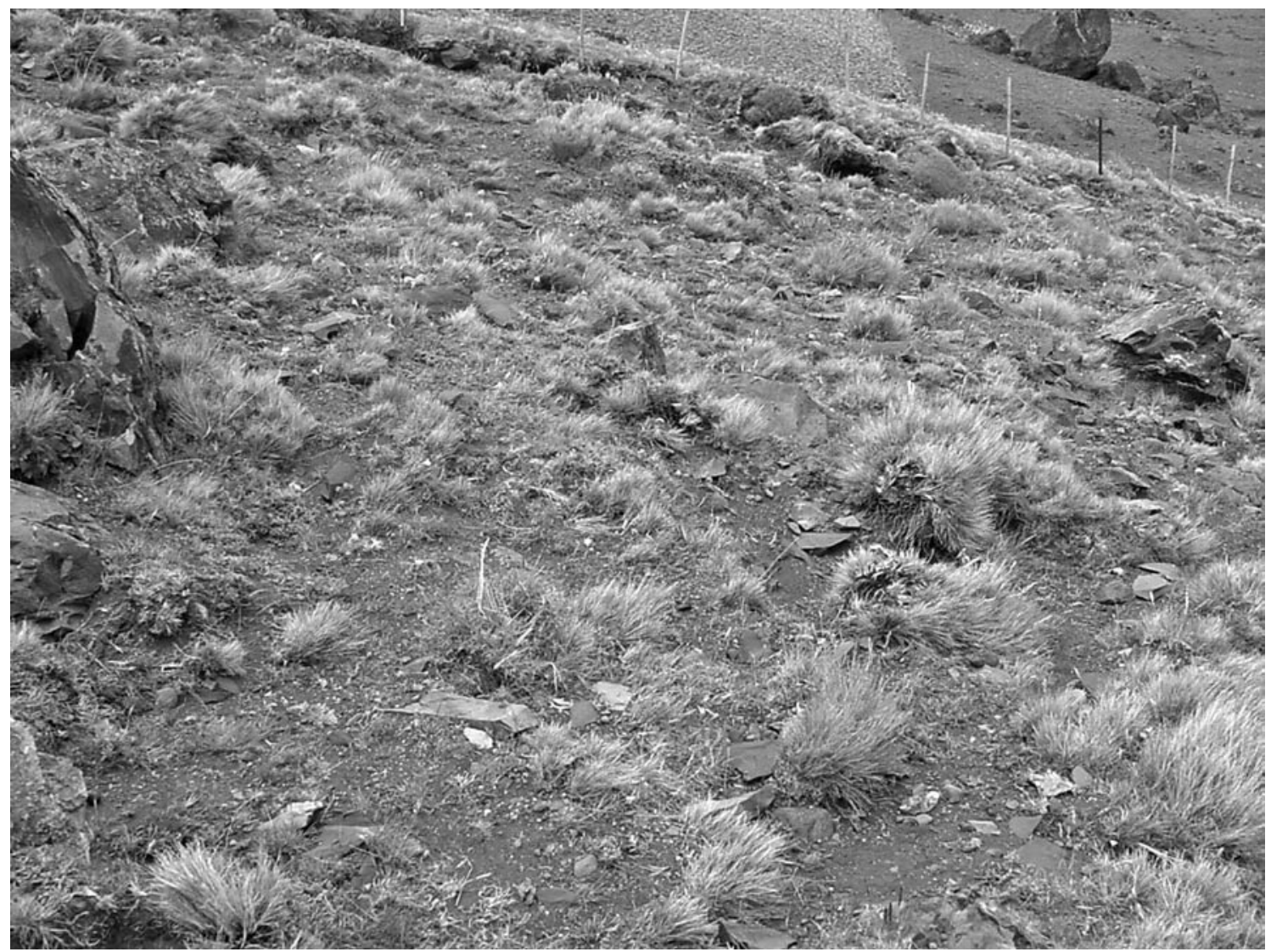

Fig. 8. Fuente de RGFO en el talud de Cerro Tridente.

mencionadas (Borrazzo 2006a), alcanzando en el conjunto recuperado en CL3 sólo el 30\% del total de artefactos de calcedonia presentes en la muestra ${ }^{4}$. En cuanto a la presencia de calcedonias macroscópicamente similares en las muestras de superficie aquí analizadas, sólo se han identificado ejemplares en T6s donde constituyen el 66\% sobre el total de artefactos de calcedonia allí recuperados.

La segunda fuente de materia prima $\left(50^{\circ}\right.$ 50497, 72운 14133) está a unos $100 \mathrm{~m}$ al Este de la fuente de calcedonia y se ubica en el tramo más bajo del faldeo del cerro Tridente (Figura 1). Es una fuente secundaria con una distribución que va de dispersa a concentrada (sensu Nami 1986b, 1991). Ocupa una superficie de ca. 200 por 30 m (Figura 8). Las materias primas han sido identificadas microscópicamente como rocas pelíticas (fangolita y lutitas), incluidas en el grupo RGFO. Estas materias

4 Todos los artefactos de calcedonia recuperados en el sondeo 1 de CL3 (Borrazzo 2006) provienen de los $60 \mathrm{~cm}$ superiores de la estratigrafía. primas se presentan en grandes bloques poliédricos semienterrados en el sector inferior del talud del cerro. Asimismo, se registran en superficie nódulos dispersos, producto de la fragmentación de los bloques mayores. Los mismos se encuentran depositados en las inmediaciones de los bloques y/o en cárcavas incipientes paralelas o subparalelas a la dirección de la pendiente. La buena calidad para la talla y el gran tamaño (hasta $1600000 \mathrm{~mm}^{3}$ ) de esos nódulos, los constituye materiales aptos para la talla. Se han observado evidencias de talla en el lugar (núcleo de extracciones paralelas, lascas angulares, lasca de módulo laminar). Las materias primas que ofrece esta fuente son las más frecuentemente observadas en las muestras arqueológicas y se han recuperado núcleos e instrumentos confeccionados sobre formas base con características morfológicas compatibles con las de los nódulos observados en esta fuente. Una característica destacable de estos nódulos es la baja frecuencia de reserva de corteza presente en ellos y el bajo redondeamiento de su superficie 
(relacionado con la baja incidencia del transporte natural de los mismos). Otras fuentes potenciales de aprovisionamiento de este grupo de materias primas están constituidas por las morenas y el cauce del río Zanja Honda (Figura 1). En este último caso, sin embargo, se constató la disponibilidad de nódulos de tamaños inferiores a los observados en la cumbre de las morenas y en el talud del cerro Tridente, y en todos los casos se trató de rodados.

\section{Tendencias en el registro de superficie del sudeste de Baguales}

Las observaciones realizadas en los diferentes loci estudiados en la localidad permiten caracterizar el registro arqueológico de superficie en términos tafonómicos y tecnológicos.

En lo que respecta a la dinámica postdepositacional, se destaca la baja frecuencia de condiciones que favorezcan el enterramiento de materiales. Los resultados obtenidos de numerosas observaciones tafonómicas sobre huesos actuales señalaron las escasas oportunidades de sepultamiento para restos óseos en la localidad. CL2 puede señalarse como una de ellas, aparentemente favorecida por la presencia de un mallín en el pasado (Barberena com. pers. 2005). Asimismo, se detectó durante uno de los muestreos de CCL3 una concentración de cinco lascas muy pequeñas ${ }^{5}$ en unos $100 \mathrm{~cm}^{2}$ (cuatro de ellas, por similitudes macroscópicas de su materia prima, posiblemente pertenecientes a una misma masa lítica) al reparo de un arbusto de mata negra (Junellia tridens). Allí, se observó que la granulometría de la matriz era mucho más fina que la observada en promedio en el locus. Esto podría señalar el rol de las plantas en la generación de condiciones de sedimentación favorables para retener partículas artefactuales pequeñas.

Por otra parte, las observaciones sobre los artefactos arqueológicos y experimentales enfatizan la necesidad de evaluar el rol de procesos de reptación asociados a la pendiente en la configuración los patrones de distribución espacial de los materiales líticos recuperados en superficie. Se postulan como algunos de sus principales efectos sobre el registro arqueológico: a) reordenamiento por tamaño/peso;

5 Tamaños de las lascas de la concentración: $22 \times 24 \times 7 \mathrm{~mm}$, 12 × 15 × 2 mm, 10 × 6 × 2 mm y $9 \times 10$ × 2 mm (largo, ancho y espesor, respectivamente). b) remoción diferencial de tamaños; c) redepositación de artefactos en sectores ubicados abajo en la pendiente, y d) fragmentación. Los estudios actualísticos en curso deberán falsar esta hipótesis. De todos modos, esto señala la importancia de identificar e integrar en los muestreos de superficie espacios afectados y vinculados por estos procesos.

En cuanto a la información tecnológica, el análisis de las nuevas muestras indica las tendencias de uso del espacio sugeridas en trabajos anteriores para la región. Éste se caracteriza por el predominio en los conjuntos arqueológicos de materias primas líticas de disponibilidad inmediata (Franco y Borrero 2000). Entre las litologías observadas en la localidad, se destaca la disponibilidad natural en frecuencia y tamaños de las RGFO. A las fuentes ya conocidas de estas materias primas (morenas y cauce de río Zanja Honda, Figura 1) se agrega la aquí presentada, localizada al pie del cerro Tridente. Los relevamientos desarrollados en esta última permiten sostener que se trata de una fuente de materia prima que ha sido efectivamente utilizada por las poblaciones prehistóricas que habitaron la localidad. Todo apunta a que las litologías más frecuentes en los conjuntos arqueológicos son las más abundantes en el área. Esto es así a excepción de las variedades de calcedonias incoloras disponibles en las inmediaciones de la cueva La Verdadera Argentina y en los depósitos de drift, las que están escasamente representadas en las muestras.

La mayor frecuencia de RGFO observada en T6 permite sugerir el rol de la cercanía a la fuente localizada en el talud del cerro Tridente como un elemento que habría incidido directamente en la selección y uso de las materias primas líticas en ese locus. En este sentido, la forma base de los núcleos allí recuperados fue en todos los casos una lasca nodular y presentan el menor número de extracciones registrado en la muestras aquí estudiadas.

La diversidad de materias primas aumenta con el tamaño de las muestras, registrándose la presencia de todas las litologías identificadas hasta el momento (RGFO, dacitas, calcedonias -incoloras, coloreadas y con impurezas-, sílices y ópalos) en las muestras más numerosas, esto es, T6 y CCL3. La manufactura con escasa formatización (retoque irregular, una sola serie de lascados) y descarte de instrumentos enteros con filos aún activos o reactivables dan cuenta del predominio de la aplicación de 
una estrategia expeditiva. Sólo en los instrumentos confeccionados sobre calcedonias coloreadas y ópalos (no identificadas entre las materias primas disponibles en la localidad), presentes en bajas frecuencias en los conjuntos arqueológicos, se observan tamaños reducidos, embotamiento y/o agotamiento, así como también la formatización de un mayor número de filos por pieza.

Se registró la presencia de lascas de arista y hojas de gran tamaño entre los instrumentos, desechos y núcleos recuperados, lo que señala la realización completa de la secuencia de producción de hojas sobre RGFO en la localidad.

Las RGFO han sido utilizadas tanto para la extracción de lascas como de hojas. Estos dos productos fueron obtenidos mediante diferentes estrategias de desbaste de los nódulos. En este sentido, los núcleos de hojas de RGFO exhiben un grado de explotación mayor al observado en los núcleos de lascas que, por lo general, son de lascados aislados. Sin embargo, no se han encontrado núcleos agotados de ninguno de estos tipos.

Como se observa en la tabla 1 , la densidad artefactual es relativamente similar en los loci ubicados en los alrededores de CL3 (CAE, CCL3, CL2), mientras que T6, el locus más alejado y localizado en una cota más alta (ca. 600 m.s.n.m.), presenta el valor más bajo. Queda por evaluarse si este patrón de distribución espacial de densidades artefactuales señala la mayor utilización humana del sector de la pampa de cerro León. A la menor densidad artefactual de T6 se suma que la exploración superficial y subsuperficial de la cueva La Verdadera Argentina (localizada por sobre los 600 m.s.n.m., CLVA en Figura 1) no registró evidencias de ocupación humana. Esto, junto con el escaso uso de rocas de excelente calidad disponibles localmente (fuente de calcedonia), sugeriría un uso humano poco intenso del área.

Hasta el momento todo apunta a un uso marginal del espacio periférico de la sierra Baguales, donde la expeditividad habría sido la estrategia tecnológica priorizada en la utilización de materias primas de disponibilidad local. La presencia y características de artefactos confeccionados sobre materias primas de procedencia no local señala la existencia de conexiones espaciales a una escala mayor y sugiere un uso logístico del área. En el marco de esa red, el sudeste de Baguales habría sido utilizado por lapsos temporales cortos (Franco y Borrero 2000). Espacios con excelente reparo y visual (p.e. cueva La Verdadera Argentina) no presentan evidencias de haber sido utilizados. Esto, junto con las reducidas evidencias de uso de las calcedonias disponibles en la localidad y la escasa explotación observada sobre los núcleos en general, refuerza la hipótesis de una baja intensidad de ocupación. Los pocos registros de actividades de reclamación en los materiales de la muestra se restringen a núcleos de materias primas minoritarias (calcedonia en CL2 y dacita en CCL3), una de ellas de procedencia no local. Esto último, a diferencia de la tendencia observada para sitios con reparo (p.e. CL1 y CL3), apuntaría hacia una baja reocupación de los espacios a cielo abierto. Por su parte, la drástica caída en la densidad de hallazgos registrada en CL3 -uno de los sitios con evidencia de reocupación puntual- sugiere la existencia de factores estrictos de localización y la permanencia de los mismos criterios seleccionados a través del tiempo.

Por el momento, la cronología de la presencia humana en el sur de Baguales se ubica ca. 4500 años (Borrero et al. 2006, Prieto 1993-94, este trabajo). El nuevo fechado obtenido en CL3 dista unos $50 \mathrm{~cm}$ de la base de la estratigrafía fértil, lo que indica que el área ha sido utilizada, al menos, con anterioridad a ese momento. Son necesarios nuevos fechados que provean un marco cronológico más ajustado para el inicio del uso de este sector.

\section{AGRADECIMIENTOS}

A la Dra. María Clara Etchichury por las identificaciones microscópicas de cortes delgados. A Luis Alberto Borrero por la lectura crítica de versiones anteriores y las enriquecedoras discusiones sostenidas en torno a este trabajo. A mis compañeros de trabajo, Fabiana Martin y Ramiro Barberena, por la ayuda e información brindada. A la familia Sturzenbaum, al Sr. Juan Pablo Riquez y señora, y a los Sres. Trujillo, Felidor Parada Casanova y Manuel Vera por permitir y facilitar los trabajos de campo en la estancia La Verdadera Argentina. Al Sr. Alberto Bourguet, por los datos aportados. Este trabajo forma parte del proyecto "Corredores acuáticos en la estepa patagónica: un estudio micro-regional" (UBACyT F-124), financiado por la Universidad de Buenos Aires. 


\section{BIBLIOGRAFÍA CITADA}

ARAGÓN, E. y N. V. FRANCO 1997 Características de las rocas para la talla por percusión y propiedades petrográficas. Anales del Instituto de la Patagonia, Serie Cs. Humanas, 25:187-199. Punta Arenas.

ASCHERO, C. A. 1975 Ensayo para una clasificación morfológica de los artefactos líticos aplicada a estudios tipológicos comparativos. Informe presentado a CONICET. Buenos Aires. MS.

1983 Ensayo para una clasificación morfológica de los artefactos líticos aplicada a estudios tipológicos comparativos. Informe presentado a CONICET. Revisión 1983. Buenos Aires. MS.

BORRAZZO, K.B. 2007. Una cuadrícula experimental en la estepa: primeras observaciones. Trabajo presentado en el XVI Congreso Nacional de Arqueología Argentina (San Salvador de Jujuy, Pcia. de Jujuy).

2006a. Tecnología lítica del alero Cerro León 3 (Santa Cruz, Argentina). Magallania 34(2):63-74. Punta Arenas, Chile.

2006b. Tafonomía lítica en dunas: una propuesta para el análisis de los artefactos líticos. Revista Intersecciones en Antropología 7:247-261. UNCPBA, Olavaria.

2004. Hacia una tafonomía lítica. Tesis de grado para optar por el grado de Licenciada en Ciencias Antropológicas (orientación arqueológica). Facultad de Filosofía y Letras, Universidad de Buenos Aires. MS.

BORRERO, L.A. 2003. Corredores acuáticos en la estepa patagónica: un estudio micro-regional (UBACyT F-124). Proyecto presentado a la Secretaría de Ciencia y Técnica de la Universidad de Buenos Aires. MS.

BORRERO, L., R. BARBERENA, F. MARTIN Y K. BORRAZZO. 2007. Collapsed Rockshelters in Patagonia. En Proceedings of the XV World Congress UISPP (Lisbon, 4-9 September 2006) On Shelter's Ledge: Histories, Theories and Methods of Rockshelter Research Vol. 14, Session C54 editado por M. Kornfeld, S. Vasil'ev y L. Miotti., pp.135-139, BAR International, Oxford.

BORRERO, L.A., N.V. FRANCO, F.M. MARTIN, R. BARBERENA, R. GUICHON, J.B. BELARDI, C. FAVIER DUBOIS, L. L'HEUREUX. 2006. Las Cabeceras del Coyle: información arqueológica y circulación de poblaciones humanas. En: Pasado y Presente en la cuenca del río Coyle, editado por Carballo Marina, F., J. Belardi y S. Espinosa, pp. 75-95. Universidad Nacional de la Patagonia Austral, Unidad Académica de Río Gallegos. Río Gallegos.
CIVALERO M.T. Y N.V. FRANCO. 2003. Early human occupations in Western Santa Cruz Province, Saouthernmost South America. Quaternary Interantional 109-110:77-86.

CHARLIN, J. 2005. Utilización de materias primas líticas en el campo volcánico Pali Aike (Pcia. de Santa Cruz, Argentina). Una primera aproximación a partir del análisis de núcleos. Revista Werken 7:39-55, Santiago.

FRANCO, N. V. 2002 Estrategias de utilización de recursos líticos en la cuenca superior del río Santa Cruz (Argentina). Tesis doctoral. Facultad de Filosofía y Letras, Universidad de Buenos Aires. MS.

FRANCO, N.V. y E. ARAGÓN 2004. Variabilidad en fuentes secundarias de aprovisionamiento lítico: El caso del sur del lago Argentino (Santa Cruz, Argentina). Estudios Atacameños 28:71-85. San Pedro de Atacama.

FRANCO, N.V. y L.A. BORRERO 2000. Estrategias de utilización de sierra Baguales. Contribución Arqueológica $N^{\circ} 5$. Museo Regional de Atacama. Actas del XIV Congreso Nacional de Arqueología Chilena, editado por la Sociedad Chilena de Arqueología, DIBAM y Museo Regional de Atacama, pp. 269-283. Copiapó.

FRANCO, N.V., L.A. BORRERO, J.B. BELARDI, F. CARBALLO MARINA, F.M. MARTIN, P. CAMPAN, C. FAVIER DUBOIS, N. STADLER, M.I. HERNÁNDEZ LLOSAS, H. CEPEDA, A.S. MUÑOZ, F. BORELLA, F. MUÑOZ e I. CRUZ 1999. Arqueología del cordón Baguales y sistema lacustre al sur del lago Argentino. Præehistoria 3:65-86. Buenos Aires.

FRANCO, N.V., L.A. BORRERO Y M.V. MANCINI 2004. Environmental changes and hunter-gatherers in southern Patagonia: Lago Argentino and Cabo Virgenes (Argentina). Before Farming 2004/3, 17 pp.

HAUTHAL, R. 1899. Reseña de los hallazgos en las cavernas de Última Esperanza. Revista del Museo de la Plata IX:409-420.

HISCOCK, P. 2002. Quantifying the size of artefact assemblages. Journal of Archaeological Science 29:251-258.

KUHN, S. 2004. Upper Paleolithic raw material economies at Üça izli cave, Turkey. Journal of Anthropological Archaeology 23:431-448.

MASSONE, M. y A. PRIETO. 2004. Evaluación de la modalidad Fell 1 en Magallanes. Chungará, Revista Chilena de Antropología, Volumen Especial, pp.303-315, Arica.

MELTZER, D.J. 1989. Was Stone Exchanged Among Eastern North American Paleoindians? En Eastern Paleoindian lithic resource use, C.J. Ellis y J. Lothrop (ed), pp. 1139. Westview Press, Boulder. 
MORANO, S., A. GONZÁLEZ Y A. IGLESIAS 2008. Rescate del "Chenque" del Cerro Guido. Trabajo presentado en las VII Jornadas de Arquelogía de la Patagonia, Ushuaia.

NAMI, H. 1986a. Excavación arqueológica y hallazgo de una punta de proyectil FELL 1 en la Cueva del Medio, Seno de Última Esperanza, Chile. Anales del Instituto de la Patagonia, Serie Cs. Humanas, 16:103-109.

1986b. Experimentos para el estudio de la tecnología bifacial de las ocupaciones tardías en el extremo sur de la $\mathrm{Pa}$ tagonia Continental. PREP: Informes de Investigación 5:1- 120. Buenos Aires.

1987. Cueva del Medio: Perspectivas arqueológicas para la Patagonia Austral. Anales del Instituto de la Patagonia, Serie Cs. Humanas, 17:73-106.

1991. El subsistema tecnológico de confección de instrumentos líticos y explotación de los recursos del ambiente: Una nueva vía de aproximación. Shincal 2:33-53.

ORTIZ TRONCOSO, O. 1972. Material lítico de Patagonia Austral. Seis yacimientos de superficie. Anales del Instituto de Patagonia, Serie Cs. Humanas, 3:49-82.
PRIETO, A. 1991. Cazadores Tempranos y Tardíos en la Cueva 1 Lago Sofía. Anales del Instituto de la Patagonia, Serie Cs. Humanas, 20:75-99.

PRIETO, A. 1993-4. Algunos datos en torno a los enterratorios humanos de la región continental de Magallanes. Anales del Instituto de la Patagonia, Serie Cs. Humanas, 22:91-100.

RAMOS, M. 1993. Propuesta terminológica para la técnica arqueológica de ensamblaje. Revista de la sección Prehistoria, Arqueología 3:199-212. Instituto de Ciencias Antropológicas de la Facultad de Filosofía y Letras, UBA. Buenos Aires.

SAN ROMÁN, M y F. MORELLO

2003. Nota sobre una excavación de sondeo en el alero Cerro Castillo 1. Provincia de Última Esperanza, Magallanes, Chile. Magallania 31:139-147.

1999. Caracterización Arqueológica Preliminar de la Cuenca del río Baguales (Provincia Última Esperanza, Magallanes, Chile. Anales del Instituto de la Patagonia, Serie Cs. Humanas 27:199-208. Punta Arenas 